\title{
Experiencias alternativas de producción, comercialización y consumo de alimentos en el contexto de la pandemia COVID-19
}

Food Production, Commercialization and Consumption Alternatives Experiences in the COVID-19 Pandemic Context

Experiências alternativas de produção, comercialização e consumo de alimentos no contexto da pandemia COVID-19

\author{
Ester Schiavo* \\ Juan Carlos Travela**
}

Recibido: 20 de noviembre de 2020

Aprobado: 29 de junio de 2021

https://doi.org/10.12804/revistas.urosario.edu.co/territorios/a.9923

Para citar este artículo

Schiavo, E., \& Travela, J. C. (2022). Experiencias alternativas de producción, comercialización y consumo de alimentos en el contexto de la pandemia Covid-19. Territorios, (46), 1-17. https://doi.org/10.12804/ revistas.urosario.edu.co/territorios/a.9923

\begin{abstract}
* Instituto de Estudios sobre la Ciencia y la Tecnología (IESCT), Universidad Nacional de Quilmes. Correo electrónico: eschiavo@gmail.com. ORCID: https://orcid.org/00000003-4261-3756

** Instituto de Estudios sobre la Ciencia y la Tecnología (IESCT), Consejo Nacional de Investigaciones Cientificas y Técnicas (CONICET). Correo electrónico: juancarlostravela@hotmail.com. ORCID: https://orcid.org/00000002-7896-8121
\end{abstract}


Palabras clave

Patrón neoliberal; desarrollo territorial; rol del Estado; sistema

alimentario; crisis socioambiental;

pandemia; Unión de

Trabajadores de la

Tierra.

Keywords

Neoliberal pattern; territorial development;

Sate role; food system;

socio-environmental crisis; pandemic; Land Workers Union.

Palavras-chave

Padrão neoliberal; desenvolvimento territorial; papel

do Estado; sistema alimentar; crise socioambiental; pandemia; sindicato dos trabalhadores da terra.

territarias 46

\section{RESUMEN}

El patrón neoliberal de acumulación de capital ha afectado la conformación de las ciudades latinoamericanas, a su vez, aportó a la construcción de un modelo hegemónico de producción y acceso a los alimentos, con resultados negativos en términos socioambientales y económicos. Situación que se agrava por la crisis producto del covid-19. Ante ello, se propone identificar y analizar críticamente experiencias alternativas de producción, comercialización y consumo de alimentos, para conocer el rol que desempeñan los Estados, así como los efectos territoriales de dichas experiencias. A tal fin, se presenta el estado de la cuestión y como referencia empírica se consideraron cuatro casos en Argentina, pero se selecciona como caso de estudio solo uno de ellos, la Unión de Trabajadores de la Tierra, por ser el único que aporta estratégicamente a la modificación de las bases del modelo dominante, mediante la planeación territorial del sistema alimentario y la articulación con el Estado.

\section{ABSTRACT}

The neoliberal pattern of capital accumulation has affected the formation of Latin American cities and contributed to the construction of a hegemonic model of food production and access, with negative socio-environmental and economic results. Situation that is aggravated by the COVID-19 crisis. In view of this, it is proposes to identify and analyse alternative experiences of food production, commercialization and consumption, in order to understand the role played by the states, as well as the territorial effects of these experiences. To this end, the state of the question is presented and as an empirical reference, four cases in Argentina were considered, but only one of them was selected as a case study, the Union de Trabajadores de la Tierra, because it is the only one that contributes strategically to modify the bases of the dominant model through the territorial planning of the food system and the articulation with the State.

\section{RESUMO}

O padrão neoliberal de acumulação de capital afetou a conformação das cidades latino-americanas, por sua vez, contribuiu para a construção de um modelo hegemônico de produção e acesso aos alimentos, com resultados negativos em termos socioambientais e econômicos. Situação que é agravada pela crise provocada pela COVID-19. Diante disso, propõe-se identificar e analisar criticamente experiências alternativas de produção, comercialização e consumo de alimentos, a fim de conhecer o papel desempenhado pelos estados, bem como os efeitos territoriais dessas experiências. Para tanto, é apresentado o estado da questão e quatro casos na Argentina foram considerados como referência empírica, mas apenas um deles, o Sindicato dos Trabalhadores da Terra, é selecionado como estudo de caso, por ser o único que estrategicamente contribui para modificar as bases do modelo dominante por meio do planejamento territorial do sistema alimentar e da articulação com o Estado. 


\section{Presentación del problema y objetivos del trabajo}

Las ciudades latinoamericanas actuales están conformadas por espacios mutuamente excluyentes, resultantes de la fragmentación física y la exclusión social, que a su vez son producto de la implementación, durante las últimas décadas, de políticas públicas basadas en el patrón neoliberal de acumulación de capital.

En este proceso se destaca el protagonismo del sector financiero asociado al mercado inmobiliario, promoviendo importantes proyectos habitacionales, recreativos, de servicios y consumos diversos orientados a sectores de alta renta. Al mismo tiempo se consolidaron grandes cadenas de hipermercados y shoppings como espacios privilegiados para el acceso a alimentos y consumos básicos.

De este modo se han ido profundizando las desigualdades preexistentes. Lo que también se traduce en la profundización de las características del régimen alimentario, causante de impactos sociales $\mathrm{y}$ ambientales negativos.

En dicho contexto, a principios del año 2020 se declara una pandemia debido a la difusión del covid-19, la cual, entre otros aspectos, trajo consigo nuevas fronteras territoriales, tanto materiales como simbólicas, dado que los distintos grados de aislamiento social impuestos por los gobiernos para combatirla, obligan a permanecer en las viviendas individuales, para quienes las poseen, $\mathrm{o}$ al interior de los barrios populares, para quienes carecen de suficiente espacio privado. En dichas condiciones, los que además cuentan con acceso a las tecnologías digitales y tienen capacidad de pago, a diferencia de quienes no reúnen estas condiciones, encuentran diversas formas de acceder a los alimentos y mantener el aislamiento social.

Pero el problema es algo más complejo dado que la seguridad alimentaria es un desafío mayor para sociedades altamente urbanizadas. Tales son los casos de Argentina, Brasil y Uruguay en la región, que además poseen grandes ciudades, pues en los sistemas alimentarios modernos, la distancia física entre productores y consumidores incrementa el número de eslabones de las cadenas que los vinculan, situación que aumenta su vulnerabilidad.

Aquí se plantea hipotéticamente que la debacle económica y social provocada por la actual pandemia brinda a su vez una oportunidad para fortalecer el rol del Estado y ampliar la base social de las alianzas con el sector privado y la sociedad civil.

En este marco, el objetivo del presente trabajo es identificar y analizar críticamente experiencias que promuevan formas alternativas de producción, comercialización y consumo de alimentos, para conocer el rol que desempeñan los Estados, así como los efectos territoriales de dichas experiencias.

A tal fin, se presenta el estado de la cuestión y como referencia empírica se consideran cuatro casos en Argentina. territarias 46 
Los que han sido identificados por ser formas alternativas a las dominantes de producción, comercialización o consumo de alimentos, y por estar promovidos por distintos sectores de la sociedad, donde predominan las organizaciones y movimientos sociales.

No obstante, se selecciona como caso de estudio tan solo uno de ellos, por ser el único que aporta estratégicamente a la planeación territorial del sistema alimentario y a la articulación con el Estado, en sus distintos niveles. Se trata de la organización social Unión de Trabajadores de la Tierra, que trabaja desde hace más de cinco años en la creación de colonias agroecológicas, es decir, asentamientos humanos dedicados a la agroecología, de las cuales ya posee seis en funcionamiento en tres provincias argentinas. Se destaca la experiencia recientemente iniciada en la localidad de Tapalqué, provincia de Buenos Aires, dado que, por primera vez se está trabajando de manera coordinada con el Estado: a nivel nacional, que aportará las tierras fiscales; a nivel provincial, que se ocupará de construir las viviendas y brindar el servicio eléctrico; y a nivel local, que tiene a su cargo la coordinación y promoción de la experiencia.

La otra hipótesis de trabajo sostiene que estas experiencias han contribuido a sobrellevar los efectos negativos de la pandemia y son positivas en tanto promueven formas alternativas de producción, comercialización y consumo de alimentos. Sin embargo, aún se encuentran en una etapa incipiente para disputar la hegemonía del actual régimen alimentario corporativo, cometido que no podrá avanzar significativamente sin una fuerte transformación del Estado.

\section{Formas dominantes de producción, comercialización y consumo de alimentos en las ciudades latinoamericanas}

El concepto de urbanismo de mercado permite dar cuenta de la manera en que se han desarrollado las ciudades latinoamericanas en las últimas décadas. En cada acción, este modelo de urbanización reconstituye relaciones a distintas escalas entre los actores institucionales y económicos involucrados, que son principalmente los gobiernos locales y el sector financiero articulado al sector inmobiliario y así, sustituye lógicas regulatorias por lógicas competitivas (Schiavo \& Gelfuso, 2018).

El urbanismo de mercado se manifiesta, entre otros aspectos: en el mayor protagonismo del sector terciario y de servicios especializados; en el rol predominante de los gobiernos locales, quienes impulsan legislaciones urbanísticas permisivas, promueven grandes desarrollos urbanos y centralidades excluyentes; y en la importación de políticas urbanas originadas en países hegemónicos u organismos multilaterales, mediante acuerdos comerciales y normas ajenas a los contextos de cada ciudad. De este 
modo, se han signado progresivamente ciudades latinoamericanas caracterizadas por la fragmentación física y la exclusión social, dando como resultado la producción de espacios mutuamente excluyentes (Schiavo \& Travela, 2019).

A su vez, a consecuencia de la expansión de la frontera urbana mediante barrios cerrados u otro tipo de grandes proyectos dirigidos a sectores de alta renta, se asiste a conflictos territoriales que tienden a expulsar a comunidades precarias de zonas periféricas. En este proceso, no solo se destaca el nuevo protagonismo del mercado inmobiliario, sino también el de las grandes cadenas de hipermercados y shoppings como espacios para el acceso a alimentos y consumos básicos, desplazando al comercio de cercanía, los servicios de proximidad y otras formas de economía social y solidaria (Schiavo et al., 2019).

Así, la profundización de las desigualdades producto de la crisis estructural del capitalismo, también se traduce en una crisis en el acceso a los alimentos.

En este sentido, la crisis alimentaria es un fenómeno cíclico característico del actual régimen alimentario corporativo (Holt-Giménez, 2009). Según Rodríguez Muñoz (2010) los regímenes alimentarios son comprendidos como periodos históricos de relativa estabilidad en las relaciones internacionales de poder y propiedad que configuran el ordenamiento de la economía agroalimentaria mundial, dispuesta a través de la acción combinada de las estrategias del Estado, las corporaciones y la población. Como se explicará en las secciones siguientes, sus implicancias socioambientales son considerables siendo el neoliberalismo el denominador común entre el régimen alimentario corporativo y el urbanismo de mercado.

En síntesis, la cadena de valor de los alimentos en las metrópolis atraviesa tres nudos críticos. Ellos son:

a) la concentración y el monopolio: un puñado de empresas controla todos los eslabones de la cadena, desde la producción, el agregado de valor en origen, el transporte y la comercialización, excluyendo cualquier tipo de competencia;

b) la mayor parte de la venta y distribución en las ciudades pasa por cadenas de consumo masivo como shoppings, hipermercados y supermercados;

c) los instrumentos de crédito a productores son gestionados en su mayoría por el sistema bancario, que excluye a actores de menor peso económico como las cooperativas y las pequeñas empresas.

En dicho marco de contracción estatal y avance privado se observan ciertas culturas emergentes promovidas en su mayor parte por organizaciones y movimientos sociales, que surgen como alternativas viables para aportar soluciones a problemas urbanos, como el del acceso a los alimentos. Las ventajas de lo local y 
la cercanía, sumadas a la posible integración de tecnologías digitales, abren un campo de experimentación de nuevos repertorios de acción colectiva que vinculan problemáticas cotidianas con disputas políticas a escala de las ciudades y regiones (Schiavo et al., 2020).

\section{Efectos de la pandemia COVID-19 sobre el territorio y el sistema alimentario}

Los canales destacados por los cuales la pandemia afecta a la región se relacionan con la disminución de la actividad económica de sus principales socios comerciales, la caída de los precios de los productos primarios, la interrupción de las cadenas globales de valor, la menor demanda de servicios de turismo y la intensificación del empeoramiento de las condiciones financieras mundiales. La Comisión Económica para América Latina y el Caribe (CEPAL) pronostica una caída del PBI de la región de al menos el 5,3\% en 2020, lo cual inducirá un aumento de la pobreza, el hambre y otras formas de inseguridad alimentaria. La caída de los ingresos empeora la dieta de los hogares y de las personas que destinan un alto porcentaje de sus gastos totales a la alimentación. En estas circunstancias se suelen reemplazar alimentos más nutritivos y caros por otros más baratos y con mayor contenido de grasas saturadas, azúcar, sodio y calorías, agravando la desigualdad nutricional

\section{territarias 46}

(CEPAL, 2020a).
Sin embargo, la preocupación no radica solo en la pérdida de ingresos y sus consecuencias. Si bien la CEPAL señala que, por su propia naturaleza, el sector alimentario puede adaptarse de mejor manera a la crisis que el promedio de la economía dado que, en términos relativos, sus cadenas globales de valor son más simples y resilientes, la oferta de alimentos también puede verse afectada por la interrupción de la cadena de suministro debido a disrupciones logísticas.

Ante este punto la CEPAL identifica un problema fundamental desde la óptica de este trabajo: la seguridad alimentaria es un desafío mayor para sociedades altamente urbanizadas pues, en los sistemas alimentarios modernos, la distancia física entre productores y consumidores incrementa el número de eslabones de las cadenas que los vinculan.

Argentina, Brasil y Uruguay, los tres mayores agroexportadores de América del Sur, son también países muy urbanizados y con grandes ciudades, situación que los hace particularmente vulnerables a la disrupción de la cadena de suministro de alimentos (CEPAL, 2020a).

A su vez, la pandemia contribuyó a la profundización en extremo del modelo dominante de ciudad neoliberal, tornándola más fragmentada territorialmente y profundizando la exclusión social, lo cual va delineando nuevas fronteras urbanas que separan más aun a unos barrios de otros y a su vez, a los ciudadanos entre sí, porque las nuevas fronteras llegan a

Ester Schiavo, Juan Carlos Travela 
las puertas de las viviendas individuales, para quienes las poseen (Preciado, 2020).

Así, el confinamiento social implementado en muchos países como respuesta a la pandemia, no solo ha traído consigo nuevas fronteras materiales y simbólicas, sino que también ha cambiado las formas de acceder a los alimentos, aumentando el uso del comercio electrónico para las compras en general. Sin embargo, estas opciones son accesibles tan solo para los hogares de mayor poder adquisitivo, pues dependen de la disponibilidad de conexión a internet y de medios de pago electrónicos (CEPAL, 2020a).

En este sentido, la CEPAL (2020b) alerta que en 2019 solo el $66,7 \%$ de los habitantes de la región tenía conexión a Internet. El tercio restante tiene un acceso limitado o carece del mismo debido a su condición económica y social, en particular por su edad o localización. Además, las diferencias en la conectividad entre la zona urbana y la rural son significativas, ya que el $67 \%$ de los hogares urbanos está conectado a internet mientras que en las zonas rurales solo lo está el $23 \%$.

Por otra parte, las bajas velocidades de conexión consolidan situaciones de exclusión ya que inhabilitan el uso de soluciones digitales, no solo para el acceso a los alimentos sino también para el teletrabajo y la educación en línea. En este sentido, en junio de 2020 , en el $44 \%$ de los países de la región no se alcanzaba la velocidad de descarga que permite desarrollar varias actividades en línea simultáneamente.

En Argentina, el nivel de penetración de la banda ancha fija es sumamente desigual, ya que, mientras que en la ciudad de Buenos Aires hay más conexiones que hogares, a nivel nacional hay 13 provincias con niveles de penetración por debajo del $50 \%$ y ocho de ellas, por debajo del $40 \%$. Otro dato relevante da cuenta que, a nivel nacional, alrededor del $40 \%$ de las conexiones son de baja velocidad (CABASE, 2019).

\section{Consecuencias socioambientales del régimen alimentario corporativo}

La llamada revolución verde sentó las bases en el último cuarto del siglo $\mathrm{xx}$ para la modernización tecnológica de la producción agrícola en múltiples países (Pessoa, 2019; Gras \& Hernández, 2016). La orientación a la exportación, la gran escala y el monocultivo caracterizaron la acelerada expansión, que implicó la reconfiguración del sistema agrario tradicional contraponiéndose al modelo de agricultura familiar (Giarracca \& Teubal, 2006). La gravedad de este proceso radica en sus consecuencias socioambientales, entre las que se pueden mencionar, por un lado, la deforestación que implica la expansión de la frontera agrícola, la pérdida de biodiversidad y de servicios ecosistémicos; y por otro, la expulsión de comunidades 
de sus territorios que terminan migrando hacia asentamientos informales ubicados en las periferias de las grandes ciudades, tanto como la elevada contaminación y precios de los alimentos (Fogel Pedroso, 2019; Pessoa, 2019).

En relación con lo señalado, Giarracca \& Teubal (2006) sostienen que uno de los mayores costos de la profundización del agronegocio son sus efectos negativos sobre la democracia, en la medida que fortalece el poder político de un sector cada vez más concentrado. Como ejemplo de ello pueden citarse las amenazas de desalojo forzado e ilegal realizadas recientemente por parte del exministro de agroindustria del anterior gobierno nacional conducido por el presidente Mauricio Macri, Miguel Etchevehere, quien a su vez fue titular de la Sociedad Rural Argentina, institución representante por excelencia de los grandes productores agropecuarios y terratenientes de la región pampeana. Esta persona pretende desalojar a su propia hermana por ser promotora del Proyecto Artigas, un intento de poner en marcha legalmente una experiencia agroecológica en la provincia de Entre Ríos (Risso, 2020).

De esta forma, la producción de alimentos está inmersa en un modelo agropecuario que posee fuertes implicancias socioambientales y una disputa creciente de los actores concernidos. Entre ellos se pueden mencionar a empresas semilleras, contratistas de maquinaria agrícola, pools de siembra y fondos de inversión, grandes productores, organizaciones empresariales y pequeños y medianos productores, que en gran parte de los casos son arrendatarios (Svampa \& Viale, 2014).

En dicho contexto, se identificaron como referentes empíricos, distintas experiencias provenientes tanto de organizaciones sociales, universidades y partidos políticos, como también del sector privado. El propósito es analizar diversos emprendimientos que promueven formas alternativas de producción, distribución y consumo de alimentos que permitan transformar en parte el modelo vigente.

Entre las mismas se incluye: la Misión Anti-Inflación (M A-I) llevada adelante por Ciudad Futura, un partido político de izquierda de la provincia de Santa Fe; las iniciativas promovidas por la Incubadora Universitaria de Economía, Mercados y Finanzas Solidarias de la Universidad Nacional de Quilmes (UNQ), en la provincia de Buenos Aires; distintas experiencias consolidadas provenientes del sector privado; y el caso de la Unión de Trabajadores de la Tierra (UTT), una organización social que promueve la agroecología y lleva adelante diversas acciones políticas con resultados destacados a nivel nacional, la cual fue seleccionada como caso de estudio por su aporte a la planeación territorial y a la articulación con el conjunto de actores involucrados en el sistema alimentario.

\section{territarias 46}




\section{Experiencias alternativas de producción, comercialización y consumo de alimentos}

En la tabla 1 se pueden observar las variables analizadas que condujeron a la selección del caso de estudio, entre las experiencias identificadas.

Tabla 1. Variables consideradas para el análisis de experiencias alternativas

\begin{tabular}{|l|c|c|c|c|}
\hline \multicolumn{1}{|c|}{ Variable } & UTT & M A-I & UNQ & Privados \\
\hline $\begin{array}{l}\text { Disputa política y } \\
\text { tenencia de la tierra }\end{array}$ & $\mathrm{X}$ & $\mathrm{X}$ & & \\
\hline $\begin{array}{l}\text { Promoción de la } \\
\text { agroecología }\end{array}$ & $\mathrm{X}$ & $\mathrm{X}$ & \\
\hline $\begin{array}{l}\text { Producción } \\
\text { sostenible }\end{array}$ & $\mathrm{X}$ & $\mathrm{X}$ & $\mathrm{X}$ \\
\hline $\begin{array}{l}\text { Acceso a alimentos } \\
\text { a bajo costo }\end{array}$ & $\mathrm{X}$ & $\mathrm{X}$ & $\mathrm{X}$ & \\
\hline $\begin{array}{l}\text { Acceso sin uso de } \\
\text { tecnologías digitales }\end{array}$ & $\mathrm{X}$ & $\mathrm{X}$ & $\mathrm{X}$ & $\mathrm{X}$ \\
\hline $\begin{array}{l}\text { Transformación de } \\
\text { canales comerciales }\end{array}$ & $\mathrm{X}$ & $\mathrm{X}$ & $\mathrm{X}$ & \\
\hline $\begin{array}{l}\text { Compromiso social } \\
\text { en la pandemia }\end{array}$ & $\mathrm{X}$ & & \\
\hline
\end{tabular}

Fuente: elaboración propia.

La M A-I es una iniciativa de Ciudad Futura, vinculada a otro de los proyectos de esta organización, la cadena láctea La Resistencia, un tambo surgido tras la disputa política por la tenencia de las tierras frente a desarrolladores inmobiliarios que culminó con la ordenanza del Cuerpo
Legislativo de Rosario que prohíbe el desarrollo de nuevos barrios cerrados en esa ciudad (Schiavo et al., 2019), lo que da cuenta del compromiso con la primera variable de análisis. No obstante, la segunda y tercera variable, la promoción de la agroecología y la producción sostenible, no son aspectos destacados en esta iniciativa, como se muestra en la tabla 1 .

La M A-I tiene como objetivo reducir los eslabones de la cadena de comercialización de los productos de la canasta básica, vinculando de forma directa a productores y consumidores, con lo cual logra fortalecer los dos puntos más débiles de la cadena. En cifras, el $80 \%$ de los proveedores de este proyecto son pequeños productores, quienes permiten la compra de la totalidad de la canasta básica a precios justos, con un ahorro promedio del 20 al $30 \%$ respecto a los precios de mercado (Schiavo et al., 2020), contribuyendo a dos de las variables de análisis consideradas, el acceso a los alimentos a bajo precio y la transformación de los canales comerciales (tabla 1 ).

Su funcionamiento está basado en la creación de círculos, compuestos por dos a cinco hogares, que hacen sus compras colectivas vía web una vez al mes. Con lo cual, si bien se requiere de conectividad para su uso, bastaría con que uno de los hogares tenga acceso a tecnologías digitales para poder acceder.

En relación al compromiso social en la pandemia, Ciudad Futura continuó sosteniendo la M A-I y aumentó su frecuencia 
bajo protocolos específicos de funcionamiento. De esta forma, los consumidores pudieron abastecerse como lo hacen usualmente y además se incentivó, dada la mayor frecuencia, una mayor compra a productores y artesanos (tabla 1 ).

Otra de las experiencias que se ha seleccionado es la de la Incubadora Universitaria de Economía, Mercados y Finanzas Solidarias de la UNQ, que lleva adelante las iniciativas Mercado Territorial-Agricultura Familiar, Almacén Autogestivo y Central Cooperativa. Si bien no se encontraron elementos específicos que se relacionen con la disputa política y la tenencia de la tierra, aspecto privilegiado en este análisis, esta incubadora universitaria se propuso como algunos de sus objetivos, construir canales de comercialización que disminuyan la dependencia con los circuitos convencionales, promover el crecimiento de emprendimientos de la economía social y solidaria, y también, promover el acceso a la alimentación sana, como se observa en la tabla 1. Para ello se impulsó la conformación de una red integrada por los distintos actores del circuito económico, la colaboración de equipos universitarios y del sistema científico-técnico, como, por ejemplo, el Instituto Nacional de Tecnología Agropecuaria (INTA), encargado de acompañar la transición hacia la agroecología. De esta forma, entre las variables de análisis consideradas, contribuye a promover la agroecología, la producción sostenible, el acceso a los alimentos a bajo precio y a transformar los canales de comercialización.

Luego de tres años de funcionamiento, en el 2018 se alcanzó la comercialización de 24 mil bolsones de verduras de estación provenientes de organizaciones de productores hortícolas agroecológicos o en transición a la agroecología del cordón sur de la provincia de Buenos Aires, siendo la UTT uno de los proveedores. Además, en 2014 se creó el Almacén Autogestivo de la UNQ, un espacio de comercialización permanente en su campus que ofrece una variedad de más de 350 bienes que forman parte de la canasta básica. Esto habilita la compra sin uso de tecnologías digitales. Central Cooperativa, otra de las iniciativas de esta incubadora, es un centro de servicios para el acopio, distribución y comercialización mayorista (Chiroque Solano \& Niño, 2019).

En relación al compromiso social en la pandemia, Central Cooperativa participó como proveedor de productos alimenticios que el Municipio de Ensenada reparte a través del Sistema Alimentario Escolar (SAE). Lo cual cobró importancia en un momento en el que los establecimientos educativos se encontraban cerrados ("Equipos de la UNQ...", 2020), (tabla 1).

Por último, COECO y Tallo Verde son dos emprendimientos privados consolidados, que no presentan evidencia de tener intención de dar una disputa política relacionada con la tenencia de la tierra o la promoción de la agroecología, pero sí llevan adelante formas alternativas de 
producción de alimentos. De esta forma, si bien no presentan avances en las primeras dos variables de análisis, sí lo hacen en la producción sostenible (tabla 1).

La Cooperativa Granjera Entrerriana de Chacras Orgánicas $(\mathrm{COECO})^{1}$ es una asociación de productores granjeros dedicados a la cría de pollos pastoriles con altos estándares de calidad que destaca la producción consciente en términos ecológicos. Sin embargo, en su página web reconoce que su producción está destinada a un consumidor de altos ingresos que abona una cifra mayor a la habitual debido a la calidad y certificación del producto. La comercialización se realiza en tiendas tradicionales, lo que habilita el acceso a sus productos sin el uso de tecnologías digitales y también de forma directa a través de su página web, a la vez que las entregas se realizan en la ciudad de Buenos Aires y la zona norte del área metropolitana de Buenos Aires (AMBA). De esta forma, si bien pretende reducir eslabones en la comercialización, no lo hace en pos de ofrecer precios bajos.

Tallo Verde ${ }^{2}$ es una huerta orgánica ubicada en la localidad de Open Door, partido de Luján, provincia de Buenos Aires. Despliega su actividad en tierras orgánicas certificadas y cuenta con la certificación de la Organización Internacional Agropecuaria (OIA). Comercializa de forma mayorista y minorista a través de su página web. Aporta en la reducción de los eslabones que hacen a la cadena de comercialización, pero al igual que en la experiencia anterior, no con la intención de ofrecer productos a bajo precio.

Estos dos emprendimientos no presentan acciones de compromiso social en la pandemia.

La UTT es el único caso donde se verifican todas las variables consideradas en la tabla 1.

\section{La Unión de Trabajadores de la Tierra (UTT)}

$\mathrm{La} \mathrm{UTT}^{3}$ es una organización social que reúne a más de 10 mil familias pequeño productoras y campesinas en 15 provincias argentinas (Ciancaglini, 2018). Su objetivo es transformar las condiciones de trabajo que impone el actual régimen alimentario corporativo.

Ante esta situación, en línea con la variable que analiza la disputa política por la tenencia de la tierra, uno de los principales proyectos de la UTT es la promoción de una ley que facilite el acceso a créditos blandos para poder adquirir las tierras que habitan, lo que les permitiría retener parte del excedente que generan con su trabajo y que hoy va a la renta terrateniente. Al igual que en los años 2016 y 2018, en el 2020 ha sido presentado en el Congreso Nacional Argentino un proyecto de ley para alcanzar este fin.

La posesión de la tierra, además de brindar la posibilidad de una vida digna para las familias campesinas, también contribuye a impulsar la agroecología, ya que un sistema agroecológico requiere
${ }^{1}$ Véase https://www.coeco. com.ar

${ }^{2}$ Véase https://www.talloverde.com

3 Véase https://uniondetrabajadoresdelatierra. com.ar/ 
de una planificación a mediano plazo (Melián, 2020).

La UTT impulsa el modelo agroecológico desde 2013 y en la actualidad cuenta con 250 familias produciendo de esa forma en aproximadamente 300 hectáreas, con un sistema técnico, con producción de insumos y con un circuito comercial propio, lo que originó el Consultorio Técnico Popular (CoTePo) conformado por técnicos integrantes de las familias productoras, que genera condiciones para la contención de las necesidades técnicas, de elaboración y provisión de insumos, y de comercialización. En su página web, afirman obtener una productividad igual o superior a la que se obtiene a partir del uso del paquete tecnológico que propone el agronegocio y que desde el segundo año, la diferencia es mayor al $20 \%$ a favor del planteo agroecológico. Además, gastan un $80 \%$ menos en insumos y obtienen alimentos con mayor calidad nutritiva e inocua para la salud y el ambiente.

A su vez, en conjunto con el INTA, la UTT está avanzando en la sistematización y aplicación del Sistema de Garantías Participativas (SGP), una herramienta de certificación participativa que avala la calidad de la producción agroecológica, otorgando valor agregado a su producción. Al momento se certificaron las primeras tres quintas agroecológicas, siendo los técnicos del CoTePo los encargados de realizar la certificación (INTA, 2020).

La evaluación de los productores comterritarias 46 prende aspectos concernientes a la calidad y las condiciones de la producción en sí misma, tanto como a las condiciones de vida de los productores y sus respectivas familias. Asimismo, incluye cuestiones relacionadas con lo comercial, dado que propone que el productor sea parte de la toma de decisiones en cuanto a la fijación de valor para la producción, siempre en sintonía con los principios del comercio justo (Rofi, 2020).

En la misma línea de trabajo, a través del CoTePo se lanzó el Programa de Impulso a la Agroecología (PIA) con la entrega de miles de plantines en las quintas de productores del cordón hortícola de la ciudad de La Plata, provincia de Buenos Aires. El mismo consiste en la producción y entrega de un kit de plantines y bioinsumos en forma de crédito a familias trabajadoras de la tierra para una producción planificada y la mejora en sus cultivos. Posteriormente, las verduras agroecológicas cosechadas serán entregadas como devolución del crédito y comercializadas a precio justo a través de distintos circuitos, entre ellos los llamados Almacén UTT (Capparelli, 2020).

Otra de las líneas de acción de la UTT es la creación de colonias agroecológicas, así denominadas por el tamaño de la explotación, en tierras del Estado, que producen alimentos sanos para las localidades donde se insertan. Al momento posee seis colonias agroecológicas en las provincias de Buenos Aires, Entre Ríos y Misiones. A su vez, se espera inaugurar la séptima colonia en Tapalqué, provincia 
de Buenos Aires, cuando sea superada la pandemia del COVID-19 (Melo, 2020). Otro aspecto relevante de esta experiencia es la transformación de los canales de comercialización, ${ }^{4}$ los que suman almacenes de ramos generales, el Mercado mayorista de Avellaneda, almacenes de campo y nodos comunitarios. La UTT no solo comercializa frutas y verduras agroecológicas sino también productos de almacén y lácteos cooperativos, donde se busca que toda la cadena productiva se rija por el principio del precio justo, tanto en relación al trabajo como a los precios para las personas consumidoras.

Los almacenes de ramos generales, abiertos de lunes a sábados, se encuentran en los barrios de Almagro y Villa Devoto de la ciudad de Buenos Aires, y en las ciudades de La Plata y Monte Grande, provincia de Buenos Aires.

El Mercado de Avellaneda, en la localidad del mismo nombre, provincia de Buenos Aires, permite además la compra mayorista.

Los almacenes de campo se encuentran situados en las ciudades de San Vicente, Alejandro Korn y Domselaar, partido de San Vicente, y en la localidad de Lisandro Olmos, partido de La Plata, todos ubicados en la provincia de Buenos Aires. A su vez, existen alrededor de 150 grupos que conforman los nodos de compra comunitaria en el AMBA y en la ciudad de La Plata. Cada nodo tiene una persona coordinadora que cada semana se ocupa de recibir los pedidos de las demás personas integrantes del grupo, organizarlos y recibir los bolsones.

Un ejemplo del crecimiento político de la organización es la asunción de $\mathrm{Na}$ huel Levaggi, referente de la UTT, como presidente del Mercado Central de la ciudad de Buenos Aires en marzo de 2020, institución en la que todos los días interactúan 10 mil personas que despachan mensualmente 106 mil toneladas de frutas y verduras para 13 millones de habitantes. Por primera vez, desde su inauguración en octubre de 1984, esta institución está bajo la conducción de un joven proveniente de la agricultura familiar y los movimientos campesinos (Mardones, 2020).

Durante la pandemia, además de continuar con su labor bajo los protocolos requeridos, la UTT asistió con donaciones a comedores populares y organizaciones barriales. Solo en los primeros tres meses de la pandemia ya había alcanzado la donación de más de 70 mil kilos de frutas y verduras en todo el país ("La UTT ya donó...", 2020), lo que demuestra su compromiso social.

\section{Reflexiones finales}

El régimen alimentario corporativo, liderado en Argentina por los terratenientes de la región pampeana y los poderes concentrados antes mencionados, es funcional al patrón neoliberal de acumulación de capital y domina el sistema de producción y acceso a los alimentos con consecuencias socioambientales no solo
${ }^{4}$ Véase https://uniondetrabajadoresdelatierra. com.ar/comercializacion-2/ 
negativas, sino también crecientemente peligrosas para la vida humana y la calidad del hábitat.

No obstante, el cambio de gobierno producido en Argentina en diciembre de 2019 y la asunción del presidente Alberto Fernández, quien lidera un frente político progresista de orientación opuesta al gobierno saliente, las transformaciones en el sector analizado demoran en concretarse.

Además, no se puede dejar de mencionar que a tan solo unos meses de asumido el nuevo gobierno se declara una pandemia producto de la difusión del COVID-19, cuyas consecuencias globales son aún difíciles de prever con certeza. En dicho contexto interesa señalar algunos hechos que dan cuenta de la vitalidad que mantiene el citado poder hegemónico en el sistema alimentario.

Por una parte, la reciente aprobación de la comercialización de trigo transgénico con la participación de instituciones públicas en su desarrollo (Resolución 41 de 2020), así como la baja temporal de retenciones a la soja (Decreto 790 de 2020), lo cual se puede interpretar como decisiones del Estado nacional afines a los intereses del citado sector de poder.

Paralelamente se reitera la referencia al problema de la familia Etchevehere, destacada integrante del citado grupo de poder, que pretende transformar una desavenencia familiar en un conflicto público en el que se enfrentan los varones de la familia que defienden el modelo de agronegocios con la única hermana mujer, que promueve la producción agroecológica mediante el Proyecto Artigas en tierras indivisas, en un oscuro juicio de sucesión; lo cual es difundido por los medios masivos de comunicación funcionales a dicho poder, con mensajes tales como si se tratara de una usurpación de tierras que traerá consigo el fin de la propiedad privada.

Ante esta situación, en el contexto de la pandemia y a la luz del análisis de las experiencias alternativas seleccionadas, es posible verificar las hipótesis formuladas. En efecto, la debacle económica y social provocada por la actual pandemia brinda a su vez una oportunidad para fortalecer el rol del Estado y ampliar la base social de las alianzas con el sector privado y la sociedad civil. Las experiencias promovidas por la UNQ y la UTT dan cuenta de que este es un camino posible, que no solo contribuye a mejorar la calidad de los alimentos producidos sino también a multiplicar sus formas de comercialización, a bajar los precios y a dignificar la vida de los trabajadores agropecuarios.

A su vez, también se verifica, particularmente en el caso de la UTT, que las experiencias alternativas han contribuido a sobrellevar los efectos negativos de la pandemia y son positivas en tanto promueven formas alternativas en la producción, comercialización y consumo de los alimentos, pero aún se encuentran en una etapa incipiente para disputar las formas hegemónicas en que la sociedad produce $\mathrm{y}$ accede a sus alimentos. Cometido en 
el cual no se podrá avanzar significativamente sin una fuerte intervención, pero también y principalmente, transformación del Estado.

\section{Referencias}

Cámara argentina de internet (CABASE). (2019). Estado de Internet en Argentina y la Región. Segundo Semestre 2019. https://www.cabase.org.ar/wp-content/uploads/2019/12/CABASEInternet-Index-II-Semestre-2019.pdf Capparelli, M. (2020, 28 de julio). Por el COVID-19 y el hambre se produjo una invasión de monos en el sudeste asiático. InfoCampo. https://www.infocampo. com.ar/la-utt-lanzo-un-sistema-decreditos-para-impulsar-la-agroecologia-en-el-cordon-horticola-platense/

Chiroque Solano, H., \& Niño, L. (2019). La experiencia de la Incubadora Universitaria de Economía, Mercados y Finanzas Solidarias de la Universidad Nacional de Quilmes. Incubación de procesos para impulsar circuitos económicos solidarios. Revista Otra Economía, 12 (21), 248-260.

Ciancaglini, S. (2018). El verdurazo: la colonia agroecológica de la UTT en Jáuregui. Revista $M U$,(124). https:// lavaca.org/notas/el-verdurazo-lacolonia-agroecologica-de-la-utt-enjauregui/

Comisión Económica para América Latina y el Caribe (CEPAL). (2020a). Cómo evitar que la crisis del COVID-19 se transforme en una crisis alimentaria: acciones urgentes contra el hambre en América Latina y el Caribe. https://www.cepal.org/es/ publicaciones/45702-como-evitarque-la-crisis-covid-19-se-transformecrisis-alimentaria-acciones

Comisión Económica para América Latina y el Caribe (CEPAL). (2020b). Universalizar el acceso a las tecnologías digitales para enfrentar los efectos del COVID-19. [No. 7. Informe Especial Covid-19]. https://repositorio.cepal.org/bitstream/handle/11362/45938/4/ S2000550_es.pdf

Decreto 790 de 2020. Nomenclatura común del Mercosur. https://www. boletinoficial.gob.ar/detalleAviso/ primera/235913/20201009

Equipos de la UNQ facilitan la incorporación de alimentos cooperativos y de la economía popular en la asistencia por el COvid-19. (2020, 17 de julio). Nodal. https://www.nodal.am/2020/07/ equipos-de-la-unq-facilitan-la-incorporacion-de-alimentos-cooperativosy-de-la-economia-popular-en-la-asistencia-por-el-covid-19/

Fogel Pedroso, R. (2019). Desarraigo sin proletarización en el agro paraguayo. Íconos, revista de ciencias sociales, (63). https://doi.org/10.17141/iconos.63.2019.3423

Giarracca, N., \& Teubal, M. (2006). Democracia y neoliberalismo en el campo argentino. Una convivencia difícil. En H. Grammont (Coord.), 
La construcción de la democracia en el campo latinoamericano (pp. 69-94). Consejo Latinoamericano de Ciencias Sociales (CLACSO). http://bibliotecavirtual.clacso.org.ar/clacso/gt/2010 0926015342/2GiarraccaTeubal.pdf

Gras, C., \& Hernández, V. (2016). Radiografía del nuevo campo argentino. Del terrateniente al empresario. Siglo XXI.

Holt-Giménez, E. (2009). Crisis alimentarias, movimiento alimentario y cambio de régimen. Ecología Politica, (38), 73-79. https://www.ecologiapolitica.info/novaweb2/wp-content/ uploads/2016/03/038_Holt-Gimenez_2009.pdf

Instituto Nacional de Tecnología Agropecuaria (INTA). (2020, 11 de junio). El INTA acompaña a la UTT en su primera experiencia de aplicación de los Sistemas de Garantias Participativas (SGP). https://inta.gob.ar/noticias/ el-inta-acompana-a-la-utt-en-su-primera-experiencia-de-aplicacion-delos-sistemas-de-garantias-participativas-sgp

La UTT ya donó más de 70 mil kilos de alimentos desde el inicio de la pandemia. (2020, 14 de mayo). Ámbito. https://www.ambito.com/economia/ campo/la-utt-ya-dono-mas-70-milkilos-alimentos-el-inicio-la-pandemia-n5102660

Mardones, C. (2020, 29 de marzo). Nahuel Levaggi: "Me convocaron con

\section{tersitarias 46} la consigna: "queremos que la gente coma más barato'”. El Tiempo argentino. https://www.tiempoar. com.ar/politica/nahuel-levaggi-meconvocaron-con-la-consigna-queremos-que-la-gente-coma-mas-barato/ Melián, L. (2020, 30 de septiembre). Proponen un Plan Procrear rural. ANccom. http://anccom.sociales.uba. ar/2020/09/30/proponen-un-planprocrear-rural/

Melo, S. (2020, 12 de julio). Tapalqué: colonias agroecológicas para producir lo que se consume. El Popular. https://www.elpopular.com.ar/ nota/146520/tapalque-coloniasagroecologicas-para-producir-lo-quese-consume

Pessoa, K. (2019, febrero). De la soya hacia la agroecología: agriculturas en disputa. Letras Verdes. Revista Latinoamericana de Estudios Socioambientales, (25), 29-53. https://doi.org/10.17141/ letrasverdes.25.2019.3373

Preciado, P. (2020, 28 de marzo). Aprendiendo del virus. El pais. https:// elpais.com/elpais/2020/03/27/ opinion/1585316952_026489.html

Resolución 4l de 2020. Ministerio de agricultura, ganadería y pesca secretaría de alimentos, bioeconomía y desarrollo regional. https://www. boletinoficial.gob.ar/detalleAviso/ primera/235913/20201009

Risso, N. (2020, 21 de octubre). Los violentos audios de la patota que convocó Miguel Etchevehere para desalojar el campo de su hermana Dolores. Página 12. https://www.paginal2.com.

Ester Schiavo, Juan Carlos Travela 
ar/300696-los-violentos-audios-dela-patota-que-convoco-miguel-etcheve Rodríguez Muñoz, F. (2010, diciembre) Regímenes, sistema y crisis agroalimentaria. El otro derecho, (42), 45-74. http://bibliotecavirtual.clacso.org.ar/ Colombia/ilsa/20120710062410/2. pdf

Rofi, D. (2020, 27 de julio). Alimentos. La UTT lanzó su programa de certificación de buenas prácticas agroecológicas. La Nación. https://www. lanacion.com.ar/economia/campo/ alimentos-la-utt-lanzo-su-programacertificacion-nid2405224/

Schiavo, E., \& Gelfuso, A. (2018). Urbanismo de mercado. Las ciudades latinoamericanas y el neoliberalismo realmente existente. Cadernos Metropole, 20(42), 423-442.

Schiavo, E., \& Travela, J. C. (2019). Estilos de desarrollo realmente existentes y disparidades territoriales en Latinoamérica y el Caribe. En L. Cuervo \& M. Délano (Eds.) Planificación multiescalar. Las desigualdades territoriales (Vol. II, pp. 17-25). Comisión Económica para América Latina y el Caribe (CEPAL).
Schiavo, E., Gelfuso, A., \& Travela, J. C. (2020). Apropiación de tecnologías digitales para el acceso a los alimentos en el contexto de la crisis global del COVID-19. La Misión Anti-Inflación en Rosario, Argentina. En R. CanalesReyes \& C. Herrera Caravajal (Cords.), Acceso, democracia y comunidades virtuales: apropiación de tecnologías digitales desde el Cono Sur (pp. 235-254). Red de Investigadores sobre Apropiación de Tecnologías Digitales (RIAT); Universidad de Los Lagos; Consejo Latinoamericano de Ciencias Sociales (CLACSO). http://biblioteca.clacso. edu.ar/clacso/se/20201125054915/ Canales-Herrera.pdf

Schiavo, E., Travela, J. C., \& Gelfuso, A. (2019, 16 al 18 de septiembre) Economia social y solidaria en el desarrollo territorial de la ciudad de Rosario. Reflexiones a partir del caso Ciudad Futura. Ponencia presentada en el II Congreso Nacional de Economía Social y Solidaria, Universidad Nacional de Quilmes, Argentina.

Svampa, M., \& Viale, E. (2014). Maldesarrollo. La Argentina del extractivismo y el despojo. Katz. territarias 46

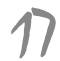

